\title{
Isotropic vector hysteresis represented by superposition of stop hysteron models
}

\author{
$\operatorname{AUTHOR}(\mathrm{S}):$ \\ Matsuo, T; Shimasaki, M
}

\section{CITATION:}

Matsuo, T ...[et al]. Isotropic vector hysteresis represented by superposition of stop hysteron models. IEEE TRANSACTIONS ON MAGNETICS 2001, 37(5): 3357-3361

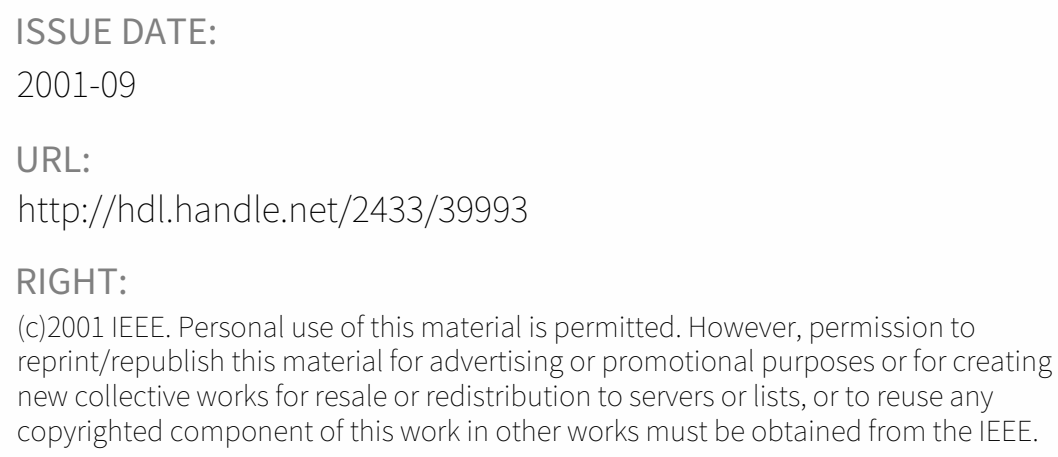




\title{
Isotropic Vector Hysteresis Represented by Superposition of Stop Hysteron Models
}

\author{
Tetsuji Matsuo, Member, IEEE and Masaaki Shimasaki, Member, IEEE
}

\begin{abstract}
The present paper first shows that the scalar stop hysteron model has the property of equal vertical chords for back-andforth input variations of the same amplitude. This property leads to an identification method of the scalar model. Secondly, identification methods are developed for the 3-D and 2-D isotropic vector models that are constructed by the superposition of scalar stop hysteron models. Numerical simulations show that these methods identify the scalar and vector models satisfactorily.
\end{abstract}

Index Terms-Equal vertical chords, identification method, isotropic vector hysteresis, stop hysteron.

\section{INTRODUCTION}

$\mathbf{T}$ HE STOP hysteron model [1]-[3] can provide the scalar hysteretic output of magnetic field $H$ from the input of magnetic flux density $B$. Since finite element analysis using magnetic vector potential requires the calculation of $H$ from $B$, this model can be an efficient tool for hysteretic electromagnetic-field analysis. The application of the stop hysteron model to electromagnetic field analysis, however, requires an efficient and precise vector hysteresis model because ferromagnetic materials have vector properties [4]-[6].

Some vector stop hysteron models, such as the geometric extension of the scalar model [1] and the superposition of scalar models [7], have been proposed. The latter is constructed in the same way as a vector Preisach model by Mayergoyz [4] and has been satisfactorily applied to 2-D eddy current analysis [7] as well as the scalar model applied to 1-D analysis [2]. The feasibility of stop hysteron models for electromagnetic field analysis, however, has not yet been sufficiently examined. In order to evaluate the representation ability of the stop hysteron models, properties of these models should be further investigated and identification methods, especially for the vector models, must be developed.

The present paper first shows that the scalar stop hysteron model has the property of equal vertical chords for back-andforth input variations of the same amplitude. This property leads to an identification method of the scalar model. Secondly, identification methods are developed for the 3-D and 2-D isotropic vector models constructed by the superposition of scalar stop hysteron models. These methods are based on integral equations similarly to those for the vector Preisach model [4], [8].

Manuscript received June 5, 2000.

The authors are with the Graduate School of Engineering, Kyoto University, Kyoto, 606-8501, Japan (e-mail: tmatsuo@kuee.kyoto-u.ac.jp and simasaki@kuee.kyoto-u.ac.jp).

Publisher Item Identifier S 0018-9464(01)07931-6.

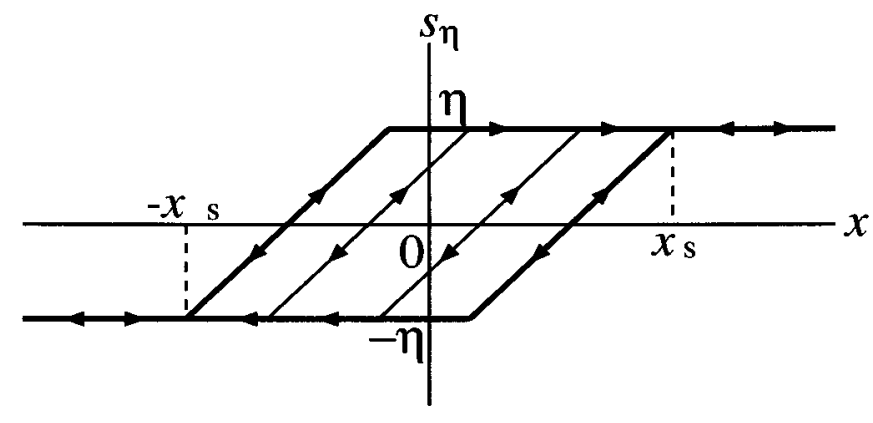

Fig. 1. Stop hysteron operator.

\section{SCALAR STOP HYSTERON MODEL}

\section{A. Property of Scalar Stop Hysteron Model}

It is assumed that a hysteretic function $y=h(x)$ exhibits its hysteretic property when $|x|<x_{\mathrm{s}}$, where $x_{\mathrm{s}}$ is a constant. The scalar stop hysteron model describes the hysteretic characteristics as

$$
y=h(x)=\int_{0}^{x_{\mathrm{s}}} g_{1}\left(\eta, s_{\eta}(x)\right) \mathrm{d} \eta
$$

where $s_{\eta}$ is the scalar stop hysteron given by (2) with height $\eta$ and $g_{1}$ is a single-valued function.

$$
s_{\eta}(x)=\left\{\begin{array}{cc}
\max \left(\min \left(x-x^{0}+s_{\eta}^{0}, \eta\right),-\eta\right) & \left(\eta<x_{\mathrm{s}}\right) \\
x & \left(\eta=x_{\mathrm{s}}\right)
\end{array}\right.
$$

where $x^{0}$ and $s_{\eta}^{0}$ are the values of $x$ and $s_{\eta}$ at the previous time-point. Since $h(x)$ becomes a single-valued function when $|x| \geq x_{\mathrm{s}}, x^{0}$ is restricted within $\left|x^{0}\right| \leq x_{\mathrm{s}}$. The characteristics of operator $s_{\eta}$ are illustrated in Fig. 1.

Equation (2) implies that $g_{1}\left(\eta, s_{\eta}(x)\right)$ becomes a single-valued function of $x$ without hysteresis when $\eta=x_{\mathrm{s}}$. The hysteretic function (1) can be accordingly divided into an irreversible part $\left(\eta<x_{\mathrm{s}}\right)$ and a reversible part $\left(\eta=x_{\mathrm{s}}\right)$ as

$$
y=h(x)=\int_{0}^{x_{\mathrm{s}}-0} g_{1}\left(\eta, s_{\eta}(x)\right) \mathrm{d} \eta+G_{1}(x)
$$

where

$$
G_{1}(x)=\int_{x_{\mathrm{s}}-0}^{x_{\mathrm{s}}} g_{1}\left(\eta, s_{\eta}(x)\right) \mathrm{d} \eta .
$$

By defining

$$
\kappa_{1}(\eta, s)=\frac{\partial g_{1}(\eta, s)}{\partial s}, \quad K_{1}(s)=\frac{\mathrm{d} G_{1}(s)}{\mathrm{d} s}
$$


3358

$h(x)$ is rewritten as

$$
\begin{aligned}
& h(x)=\int_{0}^{x_{\mathrm{s}}-0} \int_{-\eta}^{\mathrm{s}_{\eta}(x)} \kappa_{1}(\eta, s) \mathrm{d} s \mathrm{~d} \eta \\
& +\int_{-x_{\mathrm{s}}}^{x} K_{1}(s) \mathrm{d} s+h\left(-x_{\mathrm{s}}\right) .
\end{aligned}
$$

This implies that $h(x)$ is identified by determining $\kappa_{1}(\eta, s)$ and $K_{1}(s)$.

Let $h_{+}(a, x)$ and $h_{-}(a, x)$ be the outputs on ascending and descending curves, respectively, for a back-and-forth input variation given by (7) with amplitude $a\left(0 \leq a \leq x_{\mathrm{s}}\right)$ :

$$
x=u+x_{0}(-a \leq u \leq a)
$$

where $x_{0}\left(\left|x_{0}\right| \leq\left|x_{\mathrm{s}}-a\right|\right)$ is an arbitrary dc bias. It is assumed that the input $x$ has no extrema other than $x_{0} \pm a$. The hysterons of $\eta<a$ respond in a hysteretic way to this input as

$$
s_{\eta}(x)= \begin{cases}\min (u-\eta+a, \eta) & \text { (when increasing) } \\ \max (u+\eta-a,-\eta) & \text { (when decreasing). }\end{cases}
$$

On the other hand, the hysterons of $\eta \geq a$ respond assinglevalued operators for this input:

$$
s_{\eta}(x)=u+s_{\eta 0}
$$

where $s_{\eta 0}=s_{\eta}\left(x_{0}\right)$ that depends on the past history of the input prior to the back-and-forth variation.

The vertical difference between $h_{ \pm}(a, x)$ is given by

$$
\begin{aligned}
h_{+}(a, x)-h_{-}(a, x) & =\int_{0}^{a} \int_{\max (u+\eta-a,-\eta)}^{\min (u-\eta+a, \eta)} \kappa_{1}(\eta, s) \mathrm{d} s \mathrm{~d} \eta \\
& =R_{1}(\alpha, \beta) \\
R_{1}(\alpha, \beta) & =\int_{0}^{\beta} \int_{0}^{\alpha} \kappa_{1}^{\prime}\left(\alpha^{\prime}, \beta^{\prime}\right) \mathrm{d} \alpha^{\prime} \mathrm{d} \beta^{\prime}
\end{aligned}
$$

where $\alpha=(a-u) / \sqrt{2}, \beta=(a+u) / \sqrt{2}, \alpha^{\prime}=(\eta-s) / \sqrt{2}$, $\beta^{\prime}=(\eta+s) / \sqrt{2}$ (see Fig. 2(a)) and $\kappa_{1}^{\prime}(\alpha, \beta)=\kappa_{1}(a, u)$. The back-and-forth input variations of the same amplitude make equal vertical chords regardless of the dc bias $x_{0}$.

\section{B. Identification of Scalar Stop Hysteron Model}

From $(11), \kappa_{1}(a, u)$ for the irreversible part $\left(a<x_{\mathrm{s}}\right)$ is obtained as

$$
\kappa_{1}(a, u)=\kappa_{1}^{\prime}(\alpha, \beta)=\frac{\partial^{2} R_{1}}{\partial \alpha \partial \beta} .
$$

If $(\alpha+\beta) / \sqrt{2}=a>x_{\mathrm{s}}, R_{1}(\alpha, \beta)$ is defined as

$$
R_{1}(\alpha, \beta)=h_{+}\left(x_{\mathrm{s}},-x_{\mathrm{s}}+\sqrt{2} \beta\right)-h_{-}\left(x_{\mathrm{s}}, x_{\mathrm{s}}-\sqrt{2} \alpha\right)
$$

(see Fig. 2(b)). By using $R_{1}(\alpha, \beta)$ of (11) and (13), $K_{1}(s)$ for the reversible part is determined as

$$
\begin{aligned}
K_{1}(s)=\lim _{\delta \rightarrow 0} & {\left[\frac { 1 } { 2 \sqrt { 2 } \delta } \left\{R_{1}(\alpha+\delta, \beta+\delta)-R_{1}(\alpha-\delta, \beta+\delta)\right.\right.} \\
& \left.\left.-R_{1}(\alpha+\delta, \beta-\delta)+R_{1}(\alpha-\delta, \beta-\delta)\right\}\right]
\end{aligned}
$$

where $\alpha=\left(x_{\mathrm{s}}-s\right) / \sqrt{2}$ and $\beta=\left(x_{\mathrm{s}}+s\right) / \sqrt{2}$.
IEEE TRANSACTIONS ON MAGNETICS, VOL. 37, NO. 5, SEPTEMBER 2001
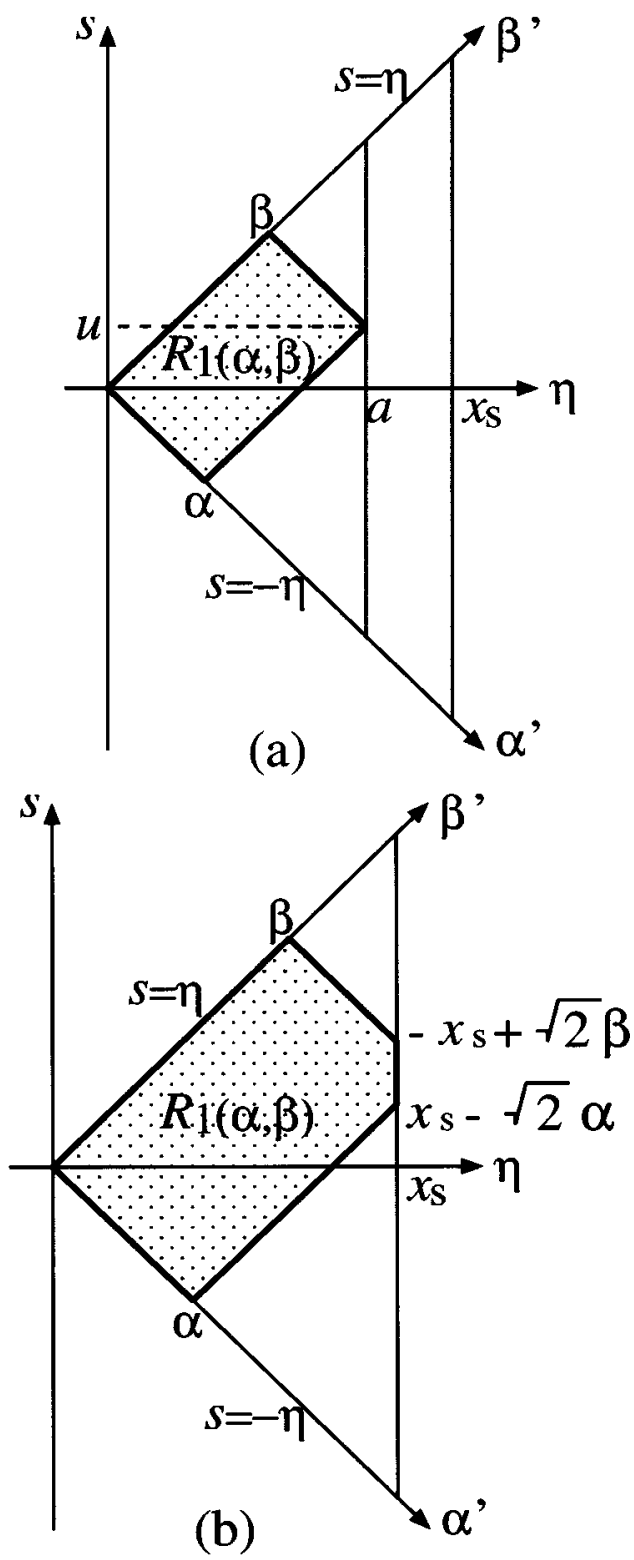

Fig. 2. Integral domain for $R_{1}$. (a) $(\alpha+\beta) / \sqrt{2} \leq x_{\mathrm{s}}$. (b) $(\alpha+\beta) / \sqrt{2}>x_{\mathrm{s}}$.

\section{Numerical Implementation of Identification Method}

The identification method can be simply implemented as follows.

The function (1) in the discretized form is written as

$$
h(x)=\sum_{k=1}^{M} \tilde{g}_{1 \eta k}\left(s_{\eta k}(x)\right)
$$

where $\eta_{k}=k x_{\mathrm{s}} / M(k=1, \ldots, M, M$ : number of hysterons $)$, $\tilde{g}_{1 \eta k}$ is a single valued function for $s_{\eta k}$. The function $\tilde{g}_{1 \eta k}$ is assumed to be piecewise linear as

$$
\begin{gathered}
\tilde{g}_{1 \eta k}(s)=\tilde{g}_{1 \eta k}\left(s_{k, j-1}\right) \\
+\tilde{\kappa}_{k, j}\left(s-s_{k, j-1}\right)\left(s_{k, j-1} \leq s \leq s_{k, j}\right)
\end{gathered}
$$

where $s_{k, j}=-\eta_{k}+j \Delta s(j=0, \ldots, k), \Delta s=2 x_{\mathrm{s}} / M$ and $\tilde{\kappa}_{k, j}=\left\{\tilde{g}_{1 \eta k}\left(s_{k, j}\right)-\tilde{g}_{1 \eta k}\left(s_{k, j-1}\right)\right\} / \Delta s$. The ascending and descending curves for the inputs (7) with the amplitudes $a=$ 
$\eta_{k}(k=1, \ldots, M)$ yield $\tilde{\kappa}_{k, j}(j=1, \ldots, k)$ as: See (17) at the bottom of the page. where: See (18) at the bottom of the page.

\section{3-D Vector STOP HYSTERON MODEL}

\section{A. Identification of 3-D Vector Stop Hysteron Model (1)}

An isotropic 3-D vector hysteresis model can be constructed as a superposition of scalar models [4].

$$
\boldsymbol{y}=\boldsymbol{h}(\boldsymbol{x})=\int_{0}^{2 \pi} \int_{0}^{\pi / 2} \boldsymbol{e}_{\theta, \varphi} h_{3}\left(\boldsymbol{e}_{\theta, \varphi} \cdot \boldsymbol{x}\right) \sin \theta \mathrm{d} \theta \mathrm{d} \varphi
$$

where $h_{3}$ is a scalar hysteretic function and $e_{\theta, \varphi}$ is the unit vector along the direction $(\theta, \varphi)$. The scalar hysteretic function $h_{3}(x)$ is given by the scalar stop hysteron model in the same way as (3) and (6) by

$$
\begin{aligned}
h_{3}(x)= & \int_{0}^{x_{\mathrm{s}}-0} g_{3}\left(\eta, s_{\eta}(x)\right) \mathrm{d} \eta+G_{3}(s) \\
= & \int_{0}^{x_{\mathrm{s}}-0} \int_{-\eta}^{\mathrm{s}_{\eta}(x)} \kappa_{3}(\eta, s) \mathrm{d} s \mathrm{~d} \eta+\int_{-x_{\mathrm{s}}}^{x} K_{3}(s) \mathrm{d} s \\
& +h_{3}\left(-x_{\mathrm{s}}\right)
\end{aligned}
$$

where

$$
\kappa_{3}(\eta, s)=\frac{\partial g_{3}(\eta, s)}{\partial s}, \quad K_{3}(s)=\frac{\mathrm{d} G_{3}(s)}{\mathrm{d} s} .
$$

When the input varies along only direction $\theta=0$, the output is given by

$$
y=h(x)=2 \pi \int_{0}^{\pi / 2} h_{3}(x \cos \theta) \cos \theta \sin \theta \mathrm{d} \theta .
$$

When this $h(x)$ has the same characteristics as the scalar function, the following equation holds.

$$
\begin{aligned}
& h_{+}(a, x)-h_{-}(a, x) \\
& =2 \pi \int_{0}^{\pi / 2}\left\{h_{3+}(a \cos \theta, x \cos \theta)\right. \\
& \left.\quad-h_{3-}(a \cos \theta, x \cos \theta)\right\} \cos \theta \sin \theta \mathrm{d} \theta
\end{aligned}
$$

where $h_{3+}(a, x)$ and $h_{3-}(a, x)$ are the values on the ascending and descending curves of $h_{3}(x)$, respectively, for the back-andforth input variation (7). Equation (23) leads to

$$
R_{1}(\alpha, \beta)=2 \pi \int_{0}^{\pi / 2} R_{3}(\alpha \cos \theta, \beta \cos \theta) \cos \theta \sin \theta \mathrm{d} \theta
$$

where $R_{3}$ is given in the same way as $R_{1}$ in (11).
Equation (24) is an integral equation for $R_{3}$, which is solved in the same way as [4]:

$$
R_{3}(\alpha, \beta)=\frac{\left\{2 R_{1}(\alpha, \beta)+\frac{\alpha \partial R_{1}}{\partial \alpha}+\frac{\beta \partial R_{1}}{\partial \beta}\right\}}{2 \pi} .
$$

Then $\kappa_{3}(a, u)$ for the irreversible part $\left(a<x_{\mathrm{s}}\right)$ is obtained as

$$
\kappa_{3}(a, u)=\kappa_{3}^{\prime}(\alpha, \beta)=\frac{\partial^{2} R_{3}(\alpha, \beta)}{\partial \alpha \partial \beta} .
$$

Equation (26) does not determine $K_{3}(s)$ for the reversible part $\left(\eta=x_{\mathrm{s}}\right)$ that is given by

$$
K_{3}(s)=\frac{\left\{3 K_{1}(s)+\frac{s \mathrm{~d} K_{1}}{\mathrm{~d} s}-x_{\mathrm{s}} \kappa_{1}\left(x_{\mathrm{s}}-0, s\right)\right\}}{2 \pi} .
$$

The derivation of (27) is given in Appendix.

\section{B. Identification of 3-D Vector Stop Hysteron Model (2)}

The back-and-forth input variation of (7) induces the reversible response of the hysterons of $\eta \geq a$. In order to discuss the relation of the reversibly responding part of the scalar and vector hysteresis models, the following function is defined.

$$
\begin{aligned}
T_{i}(a, u)= & \int_{a}^{x_{\mathrm{s}}-0} g_{i}(\eta, u) \mathrm{d} \eta+G_{i}(u) \\
= & \int_{a}^{x_{\mathrm{s}}-0} \int_{-\eta}^{u} \kappa_{i}(\eta, s) \mathrm{d} s \mathrm{~d} \eta+\int_{-x_{\mathrm{s}}}^{u} K_{i}(s) \mathrm{d} s \\
& +\int_{a}^{x_{\mathrm{s}}-0} g_{i}(\eta,-\eta) \mathrm{d} \eta+G_{i}\left(-x_{\mathrm{s}}\right)
\end{aligned}
$$

where $i=1$ or 3 . The integral domains for $\kappa_{i}$ and $K_{i}$ are shown in Fig. 3. In order to give $h(x)$ of (22) the same reversible property as the function (1) for the input (7), the following relation is required.

$$
T_{1}(\eta, s)=2 \pi \int_{0}^{\pi / 2} T_{3}(\eta \cos \theta, s \cos \theta) \cos \theta \sin \theta \mathrm{d} \theta
$$

The solution of (29) for $T_{3}$ is given by

$$
T_{3}(\eta, s)=\frac{\left\{2 T_{1}(\eta, s)+\frac{\eta \partial T_{1}}{\partial \eta}+\frac{s \partial T_{1}}{\partial s}\right\}}{2 \pi} .
$$

$$
\tilde{\kappa}_{k, j}=\frac{\left\{R_{1}^{\prime}\left(\eta_{k+1}, s_{k+1, j}\right)-R_{1}^{\prime}\left(\eta_{k}, s_{k, j}\right)-R_{1}^{\prime}\left(\eta_{k}, s_{k, j-1}\right)+R_{1}^{\prime}\left(\eta_{k-1}, s_{k-1, j-1}\right)\right\}}{\Delta s}
$$

$$
R_{1}^{\prime}\left(\eta_{k}, s_{k, j}\right)= \begin{cases}0, & (k=j=0) \\ h_{+}\left(\eta_{k}, s_{k, j}\right)-h_{-}\left(\eta_{k}, s_{k, j}\right), & (1 \leq k \leq M, 0 \leq j \leq k) \\ h_{+}\left(\eta_{M}, s_{M, j}\right)-h_{-}\left(\eta_{M}, s_{M, j-1}\right), & (k=M+1,1 \leq j \leq M)\end{cases}
$$




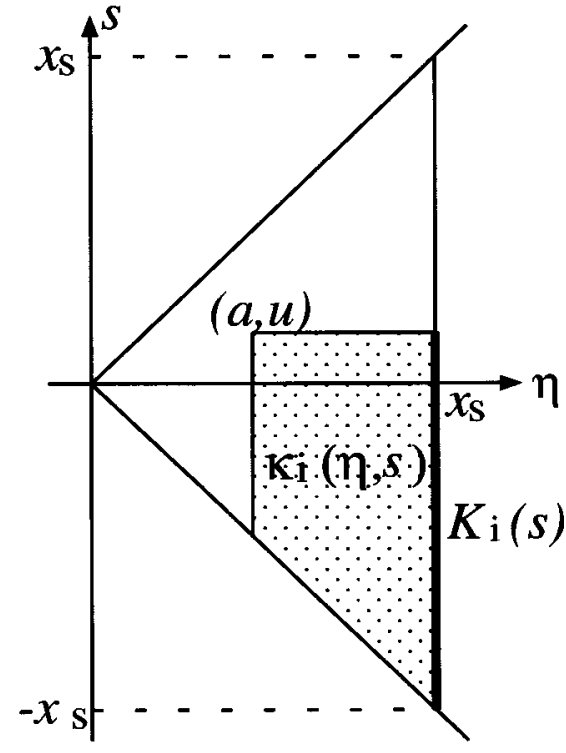

Fig. 3. Integral domain for $T_{i}$.

Functions $g_{3}\left(\eta<x_{\mathrm{s}}\right)$ and $G_{3}$ are given by (31) without using $\kappa_{3}$ and $K_{3}$.

$$
g_{3}(\eta, s)=\frac{-\partial T_{3}(\eta, s)}{\partial \eta}, \quad G_{3}(s)=T_{3}\left(x_{\mathrm{s}}-0, s\right) .
$$

If $\kappa_{3}$ and $K_{3}$ are needed, use (21) to arrive as the same $\kappa_{3}$ and $K_{3}$ as in (26) and (27) (see Appendix).

\section{2-D Vector STOP Hysteron MOdeL}

An isotropic 2-D vector hysteresis model is also constructed as a superposition of scalar models [4].

$$
\boldsymbol{y}=\boldsymbol{h}(\boldsymbol{x})=\int_{0}^{\pi} \boldsymbol{e}_{\varphi} h_{2}\left(\boldsymbol{e}_{\varphi} \cdot \boldsymbol{x}\right) \mathrm{d} \varphi
$$

where $h_{2}$ is a scalar hysteretic function and $\boldsymbol{e}_{\varphi}$ is the unit vector along the $\varphi$ direction. The scalar hysteretic function $h_{2}(x)$ is given by the scalar stop hysteron model in the same way as (3) and (20).

The unidirectional characteristics along $\varphi=0$ are given by

$$
y=h(x)=\int_{0}^{\pi} h_{2}(x \cos \varphi) \cos \varphi \mathrm{d} \varphi .
$$

This has the same characteristics as the scalar function (1) if $h_{2}(x)$ is reduced from the 3-D hysteretic function as

$$
h_{2}(x)=2 \int_{0}^{\pi / 2} h_{3}(x \sin \theta) \sin ^{2} \theta \mathrm{d} \theta
$$

where $h_{3}$ for the 3-D function is determined as in the preceding section. Equation (34) leads to

$$
T_{2}(a, u)=2 \int_{0}^{\pi / 2} T_{3}(a \sin \theta, u \sin \theta) \sin ^{2} \theta \mathrm{d} \theta
$$

where $T_{3}$ is given by (30). Functions $g_{2}$ and $G_{2}$ are given from $T_{2}$ in the same way as $g_{3}$ and $G_{3}$ are given from $T_{3}$ by (31).
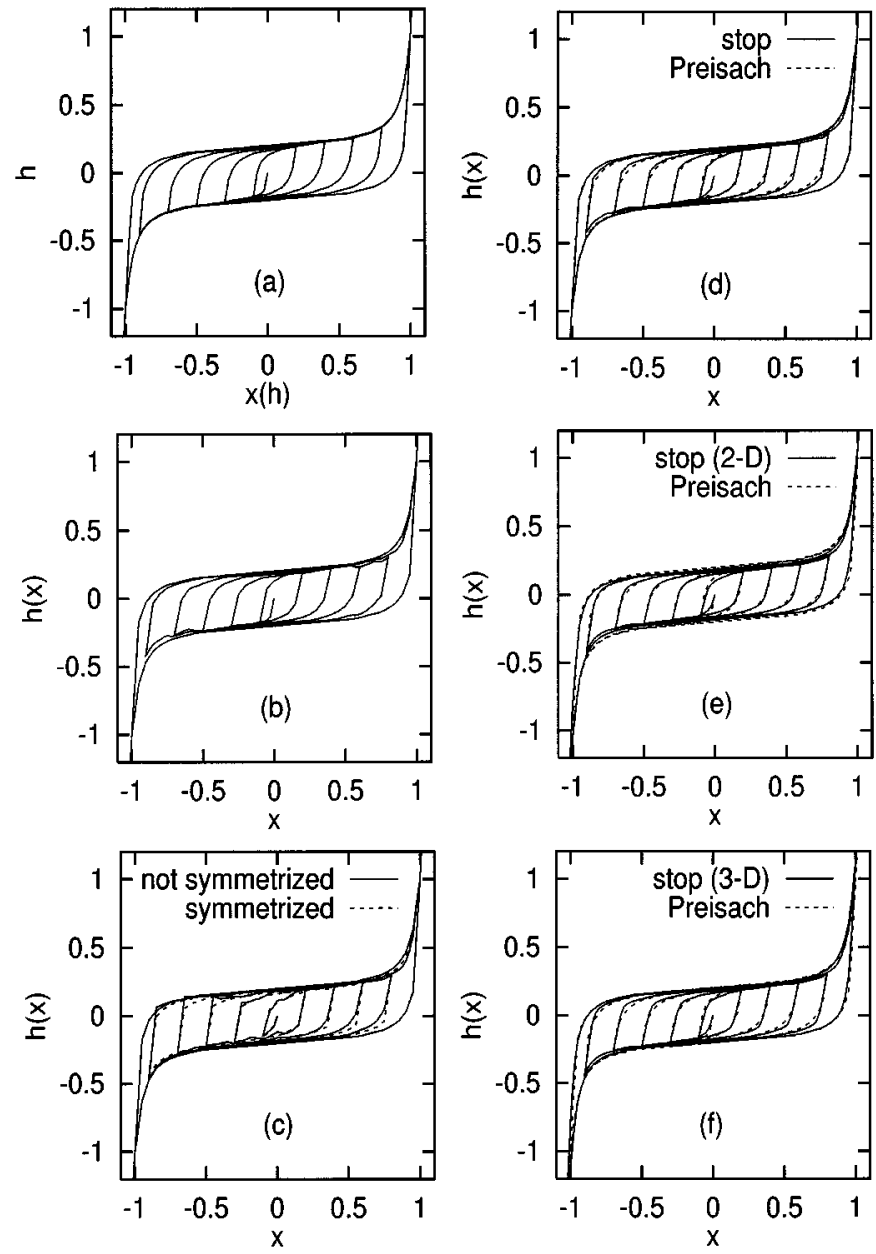

Fig. 4. Characteristics of identified stop hysteron models. (a) Preisach model, (b) Identified with (38) (a), (c) Identified with (38) (b), (d) Averaged scalar model, (e) 2-D vector model and (f) 3-D vector model.

\section{EXAMINATION OF IDENTIFICATION METHOD}

An example of hysteretic characteristics is given by the classical Preisach model [4], [6] as

$$
x(h)=0.05 h+\int_{-1}^{1} \int_{-1}^{h_{+}} \mu\left(h_{+}, h_{-}\right) \gamma_{h_{+} h_{-}} h \mathrm{~d} h_{-} \mathrm{d} h_{+}
$$

where $\mu\left(h_{+}, h_{-}\right)$is the Preisach distribution function and $\gamma_{h_{+} h_{-}}$is the elementary hysteresis operator [4]. The distribution function is set as

$$
\mu\left(h_{+}, h_{-}\right)= \begin{cases}\frac{\mu_{c}}{\left\{1+500\left(h_{+}-0.2\right)^{2}\right\}\left\{1+500\left(h_{-}+0.2\right)^{2}\right\}} \\ \left(-1 \leq h_{-} \leq h_{+} \leq 1\right) \\ 0, \quad \text { (otherwise) }\end{cases}
$$

where $\mu_{c}$ is a constant that is determined so as to set as $x( \pm 1)= \pm 1$. Fig. 4(a) shows the characteristics of $x(h)$ (inversely plotted).

The identification of the scalar stop hysteron model by (17) and (18) is performed to construct the inverse function of $x(h)$. Two types of inputs of (7) with the amplitudes $a=k x_{\mathrm{s}} / M(k=$ $\left.1, \ldots, M, x_{\mathrm{s}}=1, M=40\right)$ are examined:

(a) $x_{0}=0$, (b) $x_{0}=-x_{\mathrm{s}}+a$. 
Fig. 4(b) and (c) (solid line) show the identified characteristics from the inputs with (38) (a) and (b), respectively. These characteristics do not describe the exact inverse of the Preisach model because the inverse of Preisach model does not have the property of equal vertical chords for the inputs of the same amplitude regardless of $x_{0}$. Negative slopes $(\mathrm{d} h(x) / \mathrm{d} x<0)$ are seen in Fig. 4(b) whereas the asymmetrical inputs with (38) (b) result in the asymmetrical characteristics in Fig. 4(c). The dashed line in Fig. 4(c) shows the symmetrized characteristics that is given by symmetrizing $\tilde{\kappa}_{k, j}$. The solid line in Fig. 4(d) shows the averaged characteristics of Fig. 4(b) and (c) with symmetrization, which is given by averaging $\widetilde{\kappa}_{k, j}$. The dashed line in Fig. 4(d) is given by the Preisach model. The averaged characteristics closely approximate the inverse of the Preisach model.

From the averaged scalar model, the 3-D and 2-D vector stop hysteron models are constructed as in Fig. 4(e) and (f) by the identification method in III-B and IV. These vector models are satisfactorily identified.

\section{CONCLUSION}

It is shown that the scalar stop hysteron model has the property of equal vertical chords for back-and-forth input variations of the same amplitude with arbitrary dc bias. By using this property, an identification method for the scalar model is proposed. Identification methods, based on integral equations, are developed for 3-D and 2-D isotropic vector models. These identification methods are examined by numerical simulations, where an inverse of the Preisach model is closely approximated.

\section{APPENDIX}

The differentiation of (24) with respect to $\alpha$ and $\beta$ gives

$$
\kappa_{1}^{\prime}(\alpha, \beta)=2 \pi \int_{0}^{\pi / 2} \kappa_{3}^{\prime}(\alpha \cos \theta, \beta \cos \theta) \cos ^{3} \theta \sin \theta \mathrm{d} \theta
$$

The differentiation of (29) with respect to $s$ and $\eta$ leads to

$$
\kappa_{1}(\eta, s)=2 \pi \int_{0}^{\pi / 2} \kappa_{3}(\eta \cos \theta, s \cos \theta) \cos ^{3} \theta \sin \theta \mathrm{d} \theta
$$

The solutions of these integral equations for $\kappa_{3}$ and $\kappa_{3}^{\prime}$ are

$$
\begin{aligned}
\kappa_{3} \prime(\alpha, \beta) & =\frac{\left\{4 \kappa_{1}^{\prime}(\alpha, \beta)+\frac{\alpha \partial \kappa_{1}^{\prime}}{\partial \alpha}+\frac{\beta \partial \kappa_{1}^{\prime}}{\partial \beta}\right\}}{2 \pi} \\
& =\frac{\left\{4 \kappa_{1}(\eta, s)+\frac{\eta \partial \kappa_{1}}{\partial \eta}+\frac{s \partial \kappa_{1}}{\partial s}\right\}}{2 \pi} \\
& =\kappa_{3}(\eta, s) .
\end{aligned}
$$

The differentiation of (29) with respect to $s$ leads to

$$
T_{1 \mathrm{~s}}(\eta, s)=2 \pi \int_{0}^{\pi / 2} T_{3 \mathrm{~s}}(\eta \cos \theta, s \cos \theta) \cos ^{2} \theta \sin \theta \mathrm{d} \theta
$$

where $T_{i \mathrm{~s}}(\eta, s)=\partial T_{i}(\eta, s) / \partial s \quad(i=1,3)$. The solution of (42) for $T_{3 \mathrm{~s}}$ is given by

$$
T_{3 \mathrm{~s}}(\eta, s)=\frac{\left\{3 T_{1 \mathrm{~s}}(\eta, s)+\frac{\eta \partial T_{1 \mathrm{~s}}}{\partial \eta}+\frac{s \partial T_{1 \mathrm{~s}}}{\partial s}\right\}}{2 \pi} .
$$

By substituting $\eta=x_{\mathrm{s}}-0$ into (43), (27) is obtained.

\section{REFERENCES}

[1] M. A. Krasnosel'skii and A. V. Pokrovskii, Systems with Hysteresis. Berlin, Heidelberg: Springer-Verlag, 1989.

[2] G. Miano, C. Serpico, and C. Visone, "A new model of magnetic hysteresis, based on stop hysterons: An application to the magnetic field diffusion," IEEE Trans. Magn., vol. 32, pp. 1132-1135, 1996.

[3] S. Bobbio, G. Miano, C. Serpico, and C. Visone, "Models of magnetic hysteresis based on play and stop hysterons," IEEE Trans. Magn., vol. 33, pp. 4417-4426, 1997.

[4] I. D. Mayergoyz, Mathematical Models of Hysteresis. New York: Springer-Verlag, 1991.

[5] M. Enokizono, T. Todaka, S. Kanao, and J. Sievert, "Two-dimensional magnetic properties of silicon steel sheet subjected to a rotating field," IEEE Trans. Magn., vol. 29, pp. 3550-3552, 1993.

[6] E. D. Torre , Magnetic Hysteresis. New York: IEEE Press, 1999.

[7] T. Matsuo, Y. Osaka, and M. Shimasaki, "Eddy-current analysis using vector hysteresis models with play and stop hysterons," in Proc. COMPUMAG'99, vol. 2, Sapporo, 1999, pp. 222-223.

[8] O. Bottauscio, D. Chiarabaglio, C. Ragusa, M. Chiampi, and M. Repetto, "Analysis of isotropic materials with vector hysteresis," IEEE Trans. Magn., vol. 34, pp. 1258-1260, 1998. 